\title{
ASYMPTOTIC EQUIPARTITION OF ENERGY FOR DIFFERENTIAL EQUATIONS IN HILBERT SPACE
}

\author{
BY
}

\author{
JEROME A. GOLDSTEIN AND JAMES T. SANDEFUR, JR.
}

ABSTRACT. Of concern are second order differential equations of the form $\left(d / d t-i f_{1}(A)\right)\left(d / d t-i f_{2}(A)\right) u=0$. Here $A$ is a selfadjoint operator and $f_{1}, f_{2}$ are real-valued Borel functions on the spectrum of $A$. The Cauchy problem for this equation is governed by a certain one parameter group of unitary operators. This group allows one to define the energy of a solution; this energy depends on the initial data but not on the time $t$. The energy is broken into two parts, kinetic energy $K(t)$ and potential energy $P(t)$, and conditions on $A$, $f_{1}, f_{2}$ are given to insure asymptotic equipartition of energy: $\lim _{t \rightarrow \pm \infty} K(t)=$ $\lim _{t \rightarrow \pm \infty} P(t)$ for all choices of initial data. These results generalize the corresponding results of Goldstein for the abstract wave equation $d^{2} u / d t^{2}+A^{2} u=$

0. (In this case, $f_{1}(\lambda) \equiv \lambda, f_{2}(\lambda) \equiv-\lambda$.)

1. Introduction. Let $A$ be a selfadjoint operator on a complex Hilbert space $H$. The Cauchy problem

$$
\begin{array}{ll}
u^{\prime \prime}(t)+A^{2} u(t)=0 & (t \in \mathrm{R}=(-\infty, \infty)), \\
u(0)=f_{1} \in D\left(A^{2}\right), & u^{\prime}(0)=f_{2} \in D(A)
\end{array}
$$

is well posed; here ' $=d / d t$ and $O(A)$ denotes the domain of $A$. The energy

$$
E_{f}=\left\|u^{\prime}(t)\right\|^{2}+\|A u(t)\|^{2}
$$

depends on the data $f=\left(f_{1}, f_{2}\right)$ but not on $t$. Let

$$
K(t)=\left\|u^{\prime}(t)\right\|^{2}, \quad P(t)=\|A u(t)\|^{2}
$$

denote the kinetic and potential energy at time $t$. Goldstein [6] showed that the energy is asymptotically equipartitioned:

$$
\lim _{t \rightarrow \pm \infty} K(t)=\lim _{t \rightarrow \pm \infty} P(t)=E_{f} / 2
$$

for all choices of initial data $f$ if and only if $\lim _{t \rightarrow \pm \infty} \int_{-\infty}^{\infty} e^{i t \lambda} d\left(\left\|\Pi_{\lambda} h\right\|^{2}\right)=0$ for all $h \in H$, where $\left\{\Pi_{\lambda}: \lambda \in \mathbf{R}\right\}$ is the resolution of the identity associated with $A$, i.e., $A=\int_{-\infty}^{\infty} \lambda d \Pi_{\lambda}$ is the spectral integral representation of $A$.

Received by the editors January 6, 1975.

AMS (MOS) subject classifications (1970). Primary 47D10, 34G05; Secondary 35L15, 47B25.

Key words and phrases. Equipartition of energy, unitary group, hyperbolic equation, virial theorem. 
Now consider the more general problem

$$
v^{\prime}(t)=i B v(t) \quad(t \in \mathbf{R}), \quad v(0)=g \in O(B)
$$

in a complex Hilbert space, where $B$ is selfadjoint. (1.1), (1.2) is a special case of (1.3) (cf. [6]). The solution of (1.3) is given by $v(t)=U(t) g$, where $\{U(t)=$ $\exp (i t B): t \in \mathbf{R}\}$ is a one parameter group of unitary operators. Interpret $E_{g}=$ $\|v(t)\|^{2}$ to be the energy at time $t ; E_{g}$ depends on the data $g$ but not on $t$. The question which motivated the present paper is: With which Cauchy problems (1.3) can one decompose the energy into different types of energy and prove an asymptotic equipartition of energy theorem?

This seems to be a very difficult question, even if one specializes the context to symmetric hyperbolic systems (Friedrichs [4]) or higher order hyperbolic equations (Mizohata [8]). Mochizuki [9] , [10], generalizing [6], proved an asymptotic equipartition of energy theorem for a special class of higher order hyperbolic equations. In this paper, we generalize [6], [7] in a different way, proving asymptotic equipartition of energy for a class of second order equations of the form

$$
u^{\prime \prime}(t)+i S u^{\prime}(t)+T u(t)=0 \quad(t \in \mathbf{R})
$$

where $S$ and $T$ are certain commuting selfadjoint operators.

Other papers dealing with equipartition of energy are Bobisud and Calvert [1], Brodsky [2], Duffin [3], Glassey [5], and Shinbrot [14].

After some preliminaries in $\$ 2$, the main results are presented in $\$ \S 3$ and 4. $\$ 5$ is devoted to an example.

We wish to acknowledge some stimulating conversations on equipartition of energy with David Goldstein Costa.

2. Preliminaries. Let $H$ be a complex Hilbert space, and let $B_{1}, B_{2}$ be (not necessarily bounded) selfadjoint operators on $H$. We say that $B_{1}$ and $B_{2}$ commute if and only if $\left(\lambda i I-B_{1}\right)^{-1}$ and $\left(\mu i I-B_{2}\right)^{-1}$ commute for all $\lambda, \mu \in$ $\mathbf{R} \backslash\{0\}$ (if and only if $\exp \left(i s B_{1}\right)$ and $\exp \left(i t B_{2}\right)$ commute for all $s, t \in \mathbf{R}$ ). Also, if $B_{1}, B_{2}$ commute, there is a selfadjoint operator $A$ on $H$ and real-valued Borel functions $g_{1}, g_{2}$ defined on the spectrum of $A, \sigma(A) \subset \mathbf{R}$, such that $B_{j}=g_{j}(A)$ for $j=1,2$ (cf. [12, p. 358]).

Let $B_{1}, B_{2}$ be commuting selfadjoint operators with $B_{j}=g_{j}(A)$ as above. The equation

$$
u^{\prime \prime}+i B_{1} u^{\prime}+B_{2} u=0
$$

can be factored as

$$
\left(d / d t-i f_{1}(A)\right)\left(d / d t-i f_{2}(A)\right) u=0
$$


where $f_{1}, f_{2}$ are Borel functions on $\sigma(A)$ such that $g_{1}=f_{1}+f_{2}, g_{2}=-f_{1} f_{2}$. There are real-valued solutions $f_{1}, f_{2}$ of these equations as long as $g_{1}^{2}+4 g_{2} \geqslant 0$ on $\sigma(A)$.

Our asymptotic equipartition of energy theorem will be for the Cauchy problem (2.2). As indicated above, a great many of the equations (2.1) can be written in this form. The advantage of working with (2.2) is that one may exploit the techniques developed by Sandefur [13] to deal with the factored equation $\Pi_{j=1}^{m}\left(d / d t-C_{j}\right) u=0$.

3. The main theorem. Let $A$ be a selfadjoint operator on a complex Hilbert space $H$. Let $f_{1}, f_{2}$ be real-valued Borel functions on the spectrum $\sigma(A)$ of $A$. Let $A_{j}=i f_{j}(A)$ for $j=1,2$ and let

$$
\begin{aligned}
& D_{2}=\bigcap\left\{D\left(A_{j} A_{k}\right): 1 \leqslant j, k \leqslant 2\right\}, \\
& D_{3}=\bigcap\left\{D\left(A_{j} A_{k} A_{l}\right): 1 \leqslant j, k, l \leqslant 2\right\} ;
\end{aligned}
$$

$D_{2}$ and $D_{3}$ are dense in $H$. Define

$$
B=-2^{-1}\left(A_{1}+A_{2}\right), \quad C=2^{-1}\left(-A_{1}+A_{2}\right)
$$

with domain $O(B)=O(C)=O\left(A_{1}\right) \cap D\left(A_{2}\right)$. Note that $i B$ and $i C$ are essentially selfadjoint operators, i.e. their closures are selfadjoint.

We consider the initial value problem

$$
\begin{gathered}
\left(d / d t-A_{1}\right)\left(d / d t-A_{2}\right) u(t)=0 \quad(t \in \mathbf{R}), \\
u(0)=\phi_{1} \in D_{3}, \quad u^{\prime}(0)=\phi_{2} \in D_{2} .
\end{gathered}
$$

Theorem 3.1. Let $A, A_{1}, A_{2}$ be as above. Then for any $\phi_{1} \in D_{3}, \phi_{2} \in$ $D_{2}$, the problem (3.1), (3.2) has a unique twice continuously differentiable solution $u$. Let

$$
K(t) \equiv\left\|u^{\prime}(t)+B u(t)\right\|, \quad P(t) \equiv\|C u(t)\|^{2}, \quad t \in \mathbf{R}
$$

Then

$$
E_{\phi} \equiv K(t)+P(t)
$$

depends on $\phi$ but not on $t$. Let $\left\{\Pi_{\lambda}: \lambda \in \mathbf{R}\right\}$ be the resolution of the identity associated with $A$. Then

$$
\lim _{t \rightarrow \pm \infty} K(t)=\lim _{t \rightarrow \pm \infty} P(t)=E_{\phi} / 2
$$

for all choices of initial data $\phi$ as in (3.2) if and only if

$$
\lim _{t \rightarrow \pm \infty} \int_{-\infty}^{\infty} \exp \left\{i t\left(f_{1}(\lambda)-f_{2}(\lambda)\right)\right\} d_{\lambda}\left(\left\|\Pi_{\lambda} x\right\|^{2}\right)=0
$$

for all $x \in H$. 
When $f_{1}(t) \equiv-f_{2}(t) \equiv t,(3.1)$ reduces to (1.1), and Theorem 3.1 contains Goldstein's result [6] as a special case. [Strictly speaking more is demanded of the initial data (cf. (3.2)) than in [6], but there is no loss of generality as long as one works with a dense set of initial data. For example, it suffices to prove the theorem in [6] for initial data $\phi_{1}, \phi_{2} \in \bigcap_{n=1}^{\infty} D\left(A^{n}\right)$; the general case follows by a simple density argument.\}

With the aid of the Riemann-Lebesgue lemma it is easy to write down sufficient conditions for (3.4) to hold. For example it is sufficient to suppose $A$ is spectrally absolutely continuous and that for each $x \in H, g_{x}\left(h^{\prime}\right)^{-1} \in$ $L^{1}(h(\sigma(A)))$ where $g_{x}(\lambda)=(d / d \lambda)\left(\left\|\Pi_{\lambda} x\right\|^{2}\right)$, and where $h=f_{1}-f_{2}$ is increasing and absolutely continuous on $\sigma(A)$. We omit the simple verification (based on the change of variables $\mu=h(\lambda)$ ).

Proof of Theorem 3.1. Consider the initial value problem

$$
\frac{d}{d t}\left(\begin{array}{l}
u_{1}(t) \\
u_{2}(t)
\end{array}\right)=\left(\begin{array}{ll}
A_{1} & I \\
0 & A_{2}
\end{array}\right)\left(\begin{array}{l}
u_{1}(t) \\
u_{2}(t)
\end{array}\right) \quad(t \in \mathrm{R})
$$

in $H \oplus H$ with

$$
\left(\begin{array}{l}
u_{1}(0) \\
u_{2}(0)
\end{array}\right)=\left(\begin{array}{l}
\psi_{1} \\
\psi_{2}
\end{array}\right) \in D\left(\left(\begin{array}{ll}
A_{1} & I \\
0 & A_{2}
\end{array}\right)\right) .
$$

Since $A_{j}$ is skew-adjoint it generates a unitary group $\left\{T_{j}(t)=\exp \left(t A_{j}\right): t \in \mathbf{R}\right\}$ for $j=1,2$; therefore

$$
\left(\begin{array}{ll}
A_{1} & 0 \\
0 & A_{2}
\end{array}\right)
$$

generates the unitary group

$$
T(t)=\left\{\left(\begin{array}{lc}
T_{1}(t) & 0 \\
0 & T_{2}(t)
\end{array}\right): t \in \mathrm{R}\right\}
$$

on $H \oplus H$. Since the operator $\left(\begin{array}{ll}0 & I \\ 0 & 0\end{array}\right)$ is bounded,

$$
\left(\begin{array}{ll}
A_{1} & I \\
0 & A_{2}
\end{array}\right)=\left(\begin{array}{ll}
A_{1} & 0 \\
0 & A_{2}
\end{array}\right)+\left(\begin{array}{ll}
0 & I \\
0 & 0
\end{array}\right)
$$

generates a group $\{S(t): t \in \mathbf{R}\}$ given by

$$
S(t)\left(\begin{array}{l}
\psi_{1} \\
\psi_{2}
\end{array}\right)=\left(\begin{array}{c}
T_{1}(t) \psi_{1}+\int_{0}^{t} T_{1}(t-s) T_{2}(s) \psi_{2} d s \\
T_{2}(t) \psi_{2}
\end{array}\right)
$$


according to the Phillips' perturbation theorem [11] and a short calculation. For

$$
\psi=\left(\begin{array}{c}
\psi_{1} \\
\psi_{2}
\end{array}\right)
$$

$S(t) \psi$ is the unique twice strongly continuously differentiable solution to (3.5) and (3.6). (One can, of course, easily directly check that for $S(t) \psi$ defined by (3.7),

$$
(d / d t) S(t) \psi=\left(\begin{array}{ll}
A_{1} & I \\
0 & A_{2}
\end{array}\right) S(t) \psi
$$

$S(0) \psi=\psi$.

If we choose $\psi_{1}=\phi_{1}, \psi_{2}=\phi_{2}-A_{1} \phi_{1}$ and set

$$
\left(\begin{array}{l}
u_{1}(t) \\
u_{2}(t)
\end{array}\right)=S(t)\left(\begin{array}{l}
\psi_{1} \\
\psi_{2}
\end{array}\right), \quad t \in \mathbf{R},
$$

it follows that $u_{1}$ is a solution of (3.1), (3.2). Also by (3.2) and the definition of $D_{2}$ and $D_{3}$, we have

$$
\psi_{1}, \psi_{2} \in D\left(\left(\begin{array}{ll}
A_{1} & I \\
0 & A_{2}
\end{array}\right)^{2}\right)
$$

and so $u_{1}$ and $u_{2}=u_{1}^{\prime}-A_{1} u_{1}$ are twice strongly continuously differentiable.

If $v$ is any twice strongly continuously differentiable solution to (3.1), (3.2), then

$$
\left(\begin{array}{c}
v \\
v^{\prime}-A_{1} v
\end{array}\right)
$$

is a solution to (3.5), (3.6). But by uniqueness for (3.5), (3.6), it follows that $v=u_{1}$, and so the solution to (3.1), (3.4) is unique.

Note that

$$
\left\|u_{1}^{\prime}(t)-A_{1} u(t)\right\|^{2}=\left\|u_{2}(t)\right\|^{2}=\left\|T_{2}(t)\left(\phi_{2}-A_{1} \phi_{1}\right)\right\|^{2}=\left\|\phi_{2}-A_{1} \phi_{1}\right\|^{2}
$$

since $T_{2}(t)$ is unitary. We have the companion equality

$$
\left\|u_{1}^{\prime}(t)-A_{2} u_{1}(t)\right\|^{2}=\left\|\phi_{2}-A_{2} \phi_{1}\right\|^{2}
$$

for all $t \in \mathbf{R}$. That we can interchange the subscripts 1 and 2 in $\phi$ and $A$ follows from that fact that $\left(d / d t-A_{1}\right)\left(d / d t-A_{2}\right) u_{1}=0$ if and only if $\left(d / d t-A_{2}\right) \cdot$ $\left(d / d t-A_{1}\right) u_{1}=0$ by the closedness and commutativity of $A_{1}$ and $A_{2}$; thus the proof of (3.8) also yields (3.9). From now on let $u\left(=u_{1}\right)$ denote the unique 
solution of (3.1), (3.2). By (3.8), (3.9) and the parallelogram law,

$$
\begin{aligned}
K(t)+P(t) & =\left\|u^{\prime}(t)+B u(t)\right\|^{2}+\|C u(t)\|^{2} \\
& =2^{-1}\left\{\left\|u^{\prime}(t)+B u(t)-C u(t)\right\|^{2}+\left\|u^{\prime}(t)+B u(t)+C u(t)\right\|^{2}\right\} \\
& =2^{-1}\left\{\left\|u^{\prime}(t)-A_{2} u(t)\right\|^{2}+\left\|u^{\prime}(t)-A_{1} u(t)\right\|^{2}\right\} \\
& =2^{-1}\left\{\left\|\phi_{2}-A_{2} \phi_{1}\right\|^{2}+\left\|\phi_{2}-A_{1} \phi_{1}\right\|^{2}\right\} \\
& =\left\|\phi_{2}+B \phi_{1}\right\|^{2}+\left\|C \phi_{1}\right\|^{2}=E_{\phi}
\end{aligned}
$$

for all $t \in \mathbf{R}$. Thus the first two assertions of the theorem are proved. Next, using (3.8), (3.9) again,

$$
\begin{aligned}
P(t)= & \|C u(t)\|^{2}=4^{-1}\left\|\left(u^{\prime}(t)-A_{1} u(t)\right)-\left(u^{\prime}(t)-A_{2} u(t)\right)\right\|^{2} \\
= & 4^{-1}\left\{\left\|u^{\prime}(t)-A_{1} u(t)\right\|^{2}+\left\|u^{\prime}(t)-A_{2} u(t)\right\|^{2}\right. \\
& \left.\quad-2 \operatorname{Re}\left\langle T_{1}(t)\left(\phi_{2}-A_{2} \phi_{1}\right), T_{2}(t)\left(\phi_{2}-A_{1} \phi_{1}\right)\right\rangle\right\} \\
= & 4^{-1}\left\{\left\|\phi_{2}-A_{1} \phi_{1}\right\|^{2}+\left\|\phi_{2}-A_{2} \phi_{1}\right\|^{2}\right. \\
& \left.\quad-2 \operatorname{Re}\left\langle T_{2}(-t) T_{1}(t)\left(\phi_{2}-A_{2} \phi_{1}\right), \phi_{2}-A_{1} \phi_{1}\right\rangle\right\} \\
= & 2^{-1} E_{\phi}-\alpha(t, \phi)
\end{aligned}
$$

where

$$
\alpha(t, \phi)=2^{-1} \operatorname{Re}\left\langle T_{2}(-t) T_{1}(t)\left(\phi_{2}-A_{2} \phi_{1}\right), \phi_{2}-A_{1} \phi_{1}\right\rangle .
$$

Thus $\lim _{t \rightarrow \pm \infty} P(t)=E_{\phi} / 2$ for all $\phi$ as in (3.2) if and only if $\lim _{t \rightarrow \pm \infty} \alpha(t, \phi)=$ 0 for all such $\phi$. Taking $\phi_{1}=0$, we see that (3.3) implies

$$
\lim _{t \rightarrow \pm \infty} \operatorname{Re}\left\langle T_{2}(-t) T_{1}(t) x, x\right\rangle=0
$$

for all $x \in D_{2}$, whence for all $x \in H$. But (3.12) implies

$$
\lim _{t \rightarrow \pm \infty}\left\langle T_{2}(-t) T_{1}(t) x, y\right\rangle=0
$$

for all $x, y \in H$ by polarization. Thus (3.3) implies

$$
\lim _{t \rightarrow \pm \infty} \alpha(t, \phi)=0 \text {; }
$$

simply take $x=\phi_{2}-A_{2} \phi_{1}, y=\phi_{2}-A_{1} \phi_{1}$ in (3.13). Conversely, (3.12) implies (3.13) implies (3.14) implies (3.3); thus (3.3) and (3.12) are equivalent.

According to the spectral theorem and the associated operational calculus,

$$
\left\langle T_{2}(-t) T_{1}(t) x, x\right\rangle=\int_{-\infty}^{\infty} \exp \left\{i t\left(f_{1}(\lambda)-f_{2}(\lambda)\right)\right\} d_{\lambda}\left\langle\Pi_{\lambda} x, x\right\rangle,
$$

whence (3.12) is equivalent to (3.4) and the theorem follows. $\square$ 


\section{Asymptotic equipartition in the Cesàro sense.}

TheOREM 4.1. Let $A, A_{1}, A_{2}$ be as in Theorem 3.1. Then

$$
\lim _{\tau \rightarrow \pm \infty} \tau^{-1} \int_{0}^{\tau} K(t) d t=\lim _{\tau \rightarrow \pm \infty} \tau^{-1} \int_{0}^{\tau} P(t) d t=E_{\phi} / 2
$$

for all initial data $\phi=\left(\phi_{1}, \phi_{2}\right) \in D_{3} \times D_{2}$ if and only if

$$
\lim _{\tau \rightarrow \pm \infty} \tau^{-1} \int_{0}^{\tau} \alpha(t, \phi) d t=0
$$

for all such $\phi$ where $\alpha$ is defined by (3.11).

In particular, (4.1) holds if 0 is not an eigenvalue of $A$ (i.e. $A$ is one-to-one) and for each $\delta>0$,

$$
\inf \left\{\left|f_{1}(t)-f_{2}(t)\right|: t \in \sigma(A),|t| \geqslant \delta\right\}>0 .
$$

This generalizes a theorem in [7] in the same way that Theorem 3.1 generalized [6].

Proof of Theorem 4.1. According to (3.10), $P(t)=2^{-1} E_{\phi}-\alpha(t, \phi)$ for all initial data $\phi$. The equivalence of (4.1) and (4.2) follows easily from this and from $E_{\phi}=K(t)+P(t)$. By Theorem 3.1 it also follows that (4.2) holds if and only if

$$
\lim _{\tau \rightarrow \pm \infty} \tau^{-1} \int_{0}^{\tau}\left\langle T_{2}(-t) T_{1}(t) x, x\right\rangle d t=0
$$

for all $x \in H$. If the hypotheses of the second paragraph of the theorem hold, we have

$$
\begin{gathered}
\tau^{-1} \int_{0}^{\tau}\left\langle T_{2}(-t) T_{1}(t) \lambda, x\right\rangle d t=J_{1}+J_{2} \\
\equiv \tau^{-1} \int_{0}^{\tau} \int_{|\lambda|<\delta} \exp \left\{i t\left(f_{1}(\lambda)-f_{2}(\lambda)\right)\right\} d\left(\left\|\Pi_{\lambda} x\right\|^{2}\right) \\
+\tau^{-1} \int_{0}^{\tau} \int_{|\lambda|>\delta} \exp \left\{i t\left(f_{1}(\lambda)-f_{2}(\lambda)\right)\right\} d\left(\left\|\Pi_{\lambda} x\right\|^{2}\right) ; \\
\left|J_{1}\right| \leqslant \tau^{-1} \int_{0}^{\tau} \int_{\mid \lambda k \delta \delta} d\left(\left\|\Pi_{\lambda} x\right\|^{2}\right) \leqslant\left\|\Pi_{\delta+} x-\Pi_{\delta-} x\right\|^{2} .
\end{gathered}
$$

Given $\epsilon>0$, choose and fix $\delta>0$ such that $\left|J_{1}\right|<\epsilon / 2$; this can be done since $A$ is one-to-one. Next, if $b>0$ represents the left-hand side of (4.3) and if $h=$ $f_{1}-f_{2}$,

$$
\begin{aligned}
\left|J_{2}\right| & =\left|\int_{|\lambda|>\delta} \tau^{-1} \int_{0}^{\tau} \exp \{i \operatorname{th}(\lambda)\} d t d\left(\left\|\Pi_{\lambda} x\right\|^{2}\right)\right| \\
& =\left|\int_{|\lambda|>\delta}[\exp \{\operatorname{ith}(\lambda)\}-1][i \tau h(\lambda)]^{-1} d\left(\left\|\Pi_{\lambda} x\right\|^{2}\right)\right| \\
& \leqslant 2\|x\|^{2}|\tau b|^{-1}<\epsilon / 2
\end{aligned}
$$


if $|\tau|>4\|x\|^{2} / \epsilon b \equiv \tau(\epsilon, x)$. Consequently for each $x \in H$ and $\epsilon>0,|\tau|>$ $\tau(\epsilon, x)$ implies

$$
\left|\tau^{-1} \int_{0}^{\tau}\left\langle T_{2}(-t) T_{1}(t) x, x\right\rangle d t\right|<\epsilon,
$$

and so (4.1) holds. $\square$

If $A h=0$ for some $h \neq 0$ and if $f_{1}(0)=f_{2}(0)$, then $T_{1}(t) h=T_{2}(t) h=h$ for all real $t$, whence

$$
\tau^{-1} \int_{0}^{\tau}\left\langle T_{2}(-t) T_{1}(t) h, h\right\rangle d t=\|h\|^{2}
$$

does not tend to zero as $\tau \rightarrow \pm \infty$, hence (4.2) and (4.1) fail to hold in this case.

5. An example. The maximal operator $A$ for $i d / d x$ on the complex Hilbert space $H=L^{2}(\mathbf{R})$ is selfadjoint. Consider the initial value problem for

$$
u^{\prime \prime}(t)+P(A) u^{\prime}(t)+Q(A) u(t)=0 \quad(t \in \mathbf{R})
$$

where

$$
P(\xi)=\sum_{j=0}^{n} a_{j} \xi^{j}, \quad Q(\xi)=\sum_{j=0}^{m} b_{j} \xi^{j} / 4
$$

for $\xi \in \mathbf{R}$, where $a_{j}, b_{j} \in \mathbf{C}, a_{n} \neq 0, b_{m} \neq 0$. (Condition (3.2) in this case becomes $u(0) \in D\left(A^{3 N}\right), u^{\prime}(0) \in D\left(A^{2 N}\right)$ where $N=\max (n, m)$.) (5.1) can be rewritten in the form

$$
\left[d / d t-2^{-1}(-P(A)+R(A))\right]\left[d / d t-2^{-1}(-P(A)-R(A))\right] u=0
$$

where

$$
R(\xi)=\left\{P(\xi)^{2}-4 Q(\xi)\right\}^{1 / 2}, \quad \xi \in \mathbf{R} .
$$

Thus Theorem 3.1 (and Theorem 4.1) can be applied if $\operatorname{Re}(-P(\xi) \pm R(\xi))=0$ for each $\xi \in \mathbf{R}$. This is equivalent to:

(i) For $j=0,1, \ldots, n, a_{j}=i c_{j}$ where $c_{j} \in \mathbf{R}$;

(ii) $-\left(\sum_{j=0}^{n} c_{j} \xi^{j}\right)^{2}-\Sigma_{j=0}^{m} b_{j} \xi^{j}$ is real and nonpositive for all real $\xi$.

In particular, each $b_{j}$ must be real. We now suppose $c_{j}=-i a_{j}$ and $b_{k}$ are real for each $j, k$.

Case I. Suppose $2 n>m$. The left side of (ii) is bounded above. Consequently (ii) automatically holds if we increase $b_{0}$ enough.

Case II. Suppose $2 n=m$. If $-c_{n}^{2}-b_{2 n}<0$, the left side of (ii) is bounded above, so (ii) holds if we increase $b_{0}$ enough.

Case III. Suppose $2 n<m$. In order for the left-hand side of (ii) to be bounded above, $m$ must be even. If this is so and if $b_{m}>0$, then the conclusion of Cases I and II hold in this case as well. Thus we assume one of the following conditions: 
(a) $2 n>m$, or

(B) $2 n=m, c_{n}^{2}+b_{2 n}>0$, or

(r) $2 n<m, m$ is even, $b_{m}>0$,

and if necessary we enlarge $b_{0}$ so that (i) and (ii) hold.

Let $\left\{\Pi_{\lambda}: \lambda \in R\right.$ \} be the resolution of the identity associated with $A$ and let $\left\{\Gamma_{\mu}: \mu \geqslant 0\right\}$ be the resolution of the identity associated with the positive operator $S(A)=\left\{4 Q(A)-P(A)^{2}\right\}^{2 / 2}$. Both $A$ and $S(A)$ are spectrally absolutely continuous. Let

$$
D=\left\{x \in H: \Pi_{\lambda} x-\Pi_{-\lambda} x=\lambda \text { for some } \lambda>0\right\} .
$$

$D$ is dense in $H$. To simplify the calculation we suppose that $S(\xi)=\{4 Q(\xi)-$ $\left.P(\xi)^{2}\right\}^{1 / 2}$ is monotone decreasing on $(-\infty, L)$ and monotone increasing on $(L, \infty)$. (Without this assumption the following computations become more complicated, but the results remain valid.) For $x \in D$, choose $M$ so that $\left(\Pi_{M}-\Pi_{-M}\right) x=x$. Then

$$
\begin{aligned}
\int_{-\infty}^{\infty} \exp \left(i t\left(f_{1}(\lambda)-f_{2}(\lambda)\right)\right) d\left(\left\|\Pi_{\lambda} x\right\|^{2}\right)=\int_{-\infty}^{\infty} e^{i t S(\lambda)} d\left(\left\|\Pi_{\lambda} x\right\|^{2}\right) \\
=\int_{-M}^{L} e^{i t S(\lambda)} d\left(\left\|\Pi_{\lambda}\left(\Pi_{L} x\right)\right\|^{2}\right)+\int_{L}^{M} e^{i t S(\lambda)} d\left(\left\|\Pi_{\lambda}\left(x-\Pi_{L} x\right)\right\|^{2}\right) \\
=\int_{S(L)}^{S(-M)} e^{-i t \mu} \frac{d}{d \lambda}\left(\left\|\Pi_{\lambda}\left(\Pi_{L} x\right)\right\|^{2}\right) \frac{d}{d \mu}\left(S^{-1}(\mu)\right) d \mu \\
\quad+\int_{S(L)}^{S(M)} e^{i t \mu} \frac{d}{d \lambda}\left(\left\|\Pi_{\lambda}\left(x-\Pi_{L} x\right)\right\|^{2}\right) \frac{d}{d \mu}\left(S^{-1}(\mu)\right) d \mu \\
\rightarrow 0 \text { as } t \rightarrow \pm \infty
\end{aligned}
$$

by the Riemann-Lebesgue lemma. Since $D$ is dense, a simple density argument shows that (3.4) holds for all $x \in H$. Thus Theorem 3.1 gives asymptotic equipartition of energy for the equation

$$
\left(\partial^{2} / \partial t^{2}+P(i \partial / \partial x)(\partial / \partial t)+Q(i \partial / \partial x)\right) u(t, x)=0
$$

where

$$
P\left(i \frac{\partial}{\partial x}\right)=\sum_{j=0}^{n} i^{j+1} c_{j}\left(\frac{\partial}{\partial x}\right)^{j}, Q\left(i \frac{\partial}{\partial x}\right)=4^{-1} \cdot \sum_{j=0}^{m} i^{i} b_{j}\left(\frac{\partial}{\partial x}\right)^{j},
$$

$c_{j}, b_{k} \in \mathbf{R}, c_{n} \neq 0, b_{m} \neq 0$, and $(\alpha)$ or $(\beta)$ or $(\gamma)$ hold (and $b_{0}$ is large enough).

CONCLUDING REMARK. It would be of interest to find an equipartition of energy theorem subsuming both the result of this paper and the result of Mochizuki [9].

ADDED IN PROOF. David G. Costa has informed us that he has obtained a result similar to Theorem 3.1 . 


\section{REFERENCES}

1. L. Bobisud and J. Calvert, Energy bounds and virial theorems for abstract wave equations, Pacific J. Math. 47 (1973), 27-37. MR 48 \#4545.

2. A. R. Brodsky, On the asymptotic behavior of solutions of the wave equation, Proc. Amer. Math. Soc. 18 (1967), 207-208. MR 35 \#3289.

3. R. J. Duffin, Equipartition of energy in wave motion, J. Math. Anal. Appl. 32 (1970), 386-391. MR 42 \#4086.

4. K. O. Friedrichs, Symmetric hyperbolic linear differential equations, Comm. Pure Appl. Math. 7 (1954), 345-392. MR 16, 44.

5. R. T. Glassey, On the asymptotic behavior of nonlinear wave equations, Trans. Amer. Math. Soc. 182 (1973), 187-200. MR 48 \#9119.

6. J. A. Goldstein, An asymptotic property of solutions of wave equations, Proc. Amer. Math. Soc. 23 (1969), 359-363. MR 40 \#3365.

7. - An asymptotic property of solutions of wave equations. II, J. Math. Anal. Appl. 32 (1970), 392-399. MR 42 \#2183.

8. S. Mizohata, Quelques problèmes au bord, du type mixte, pour des équations hyperboliques, Séminaire Eq. Der. Part., Collège de France (1966-67), pp. 23-60.

9. K. Mochizuki, Asymptotic property of solutions of some higher order hyperbolic equations. I, II, Proc. Japan Acad. 46 (1970), 262-272. MR 44 \#667.

10. Spectral and scattering theory for second order elliptic differential oper. ators in an exterior domain, Univ. of Utah Lecture Notes, 1972.

11. R. S. Phillips, Perturbation theory for semi-groups of linear operators, Trans. Amer. Math. Soc. 74 (1953), 199-221. MR 14, 882.

12. F. Riesz and B. Sz.-Nagy, Leçons d'analyse fonctionnelle, Akad. Kiadó, Budapest, 1953; English transl., Functional analysis, Ungar, New York, 1955. MR 15, 132; $17,175$.

13. J. T. Sandefur, Jr., Higher order abstract Cauchy problems (to appear).

14. M. Shinbrot, Asymptotic behavior of solutions of abstract wave equations, Proc. Amer. Math. Soc. 19 (1968), 1403-1406. MR 37 \#6801.

DEPARTMENT OF MATHEMATICS, TULANE UNIVERSITY, NEW ORLEANS, LOUISIANA 70118

DEPARTMENT OF MATHEMATICS, GEORGETOWN UNIVERSITY, WASHINGTON, D. C. 20057 